\title{
Study on Mechanical Concurrent Fault Diagnosis Method Based on PSO-MRLSSVM
}

\author{
Hongtao Wang ${ }^{1}$, Lin $\mathrm{Ye}^{2}$, Chun Yan² and Hao Pan ${ }^{2, *}$ \\ ${ }^{1}$ Network Information Center, Wuhan University of Technology, Hubei Wuhan 430070, China \\ ${ }^{2}$ School of Computer Science and Technology, Wuhan University of Technology, Hubei Wuhan 430070, China \\ ${ }^{*}$ Corresponding author
}

\begin{abstract}
In view of those characteristics such as nonlinearity and high dimensional features for mechanical concurrent faults and any conventional classifier being not able to fit multi-output needs, a concurrent fault classification method based on PSO-MRLSSVM was put forward, where the simple and fast searching ability of PSO may be utilized to optimize the penalty and Kernel parameters for the MRLSSVM algorithm; thus, the aimlessness (manually specifying parameters) may be prevented and the prediction precision of MRLSSVM may be improved. Experimental results indicate that our mechanical concurrent fault diagnosis model based on PSO-MRLSSVMs works well for effective identification of concurrent fault types and diagnosis effects are good.
\end{abstract}

Keywords-mechanical faults; concurrent fault diagnosis; Particle Swarm Optimization (PSO); Multiple Regression Least Squares Support Vector Machines (Multi-regression LSSVM or MRLSSVM); parameter optimization

\section{INTRODUCTION}

Rotating machines as one kind of primary machinery components are very popular in various fields in the industrial society. Those typical rotary machines such as steam turbines, generators and pumps are essential in human society, whose are structurally made up of rotors and rotating parts. Rotors and related components (such as bearings and gears) are often out of order; especially, those high speed rotating machines such as processing and power machines easily fails. Study of fault mechanisms and diagnosis methods for rotating machines shall be of great significance for improvement of the fault diagnosis rate[1,2].

Faults may be categorized into concurrent and single faults in accordance with their occurrence cases. The former refer to two or more types of faults occurring at the same time; on the other hand, the latter refer to only one type of faults occurring at a time. Thus, the concurrent faults bring new challenges to fault diagnosis. Due to the close and cooperative relationship between equipment components, there is not a one-to-one correspondence between symptoms and causes for any fault; thus, the classification difficulty rises[3-5].

In view of characteristics (a few inputs and outputs) of any concurrent fault, the MRLSSVM was put forward here as the fault classifier. MRLSSVM developed based on LSSVM may be also utilized to solve small-sample, nonlinear and highdimension issues; in contrast, MRLSSVM may generalize multidimensional issues and minimize the overall fitting error total error of samples so that it shall be more appropriate for concurrent fault diagnosis. Because there are more unknown key parameters for MRLSSVM, artificial specification is more aimless. Thus, PSO whose search performances are better was selected here for optimization of key parameters and improvement of the classification precision for MRLSSVM. Mechanical faults were classified by means of our PSOMRLSSVM model, whose effectiveness was verified by simulation experiments.[6-8]

\section{PROFILE OF MRLSSVM AND PSO}

\section{A. Profile of MRLSSVM}

MRLSSVM put forward by Professor $\mathrm{Hu}$ Changhua is to model samples with multidimensional inputs and outputs; moreover, it is to not only minimize the total of norms of the regression weight vector of all output variables and fitting errors of the single and overall output variables but also satisfy the structural risk minimization principle. LSSVM's solution is to solve equations so that it shall need only a little time for training any multidimensional data model[9-11]. While assuming training sample set with several inputs and outputs as:

$$
\left(x_{i}, y_{i}\right), x_{i} \in R^{n}, y_{i} \in R^{k}
$$

Where: $l$ represents the number of input samples; $k$ represents the dimensions of sample outputs; and $y_{i, m}$ represents the $\mathrm{m}^{\text {th }}$ output of the $\mathrm{i}^{\text {th }}$ input.

LSSVM is generalized to k-dimension outputs, and the objective and constraint functions are as follows.

$$
\begin{aligned}
& \min \frac{1}{2} \sum_{m=1}^{k}\left\|\omega_{m}\right\|^{2}+\frac{1}{2} C_{0} \sum_{i=1}^{l} \eta_{i}^{2}+\frac{1}{2} \sum_{i=1}^{l} \sum_{m=1}^{k} C_{m} \xi_{i, m}^{2} \\
& \sum_{m=1}^{k}\left(y_{i, m}-\omega_{m}^{T} \varphi\left(x_{i}\right)-b_{m}\right)=\eta_{i} \\
& y_{i, m}-\omega_{m}^{T} \varphi\left(x_{i}\right)-b_{m}=\xi_{i, m}
\end{aligned}
$$

Where: $\mathrm{m}=1,2, \ldots, \mathrm{k} ; \mathrm{i}=1,2, \ldots, 1 ; \eta_{i}$ represents the total of the corresponding fitting errors in all dimensions of the $i^{\text {th }}$ input variable; ${ }^{\xi_{i, m}}$ represents the $\mathrm{m}^{\text {th }}$ dimension fitting error of the $\mathrm{i}^{\text {th }}$ input variable; $C_{m}$ represents the penalty parameter of the $\mathrm{m}^{\text {th }}$ dimension fitting error; and $C_{0}$ represents the penalty parameter of the total of fitting errors of all variables. 
Constraints are converted into the Lagrange form as follows:

$$
\begin{aligned}
& L=\frac{1}{2} \sum_{m=1}^{k}\left\|\omega_{m}\right\|^{2}+\frac{1}{2} \sum_{i=1}^{l} \sum_{m=1}^{k} C_{m} \xi_{i, m}^{2}+\frac{1}{2} C_{0} \sum_{i=1}^{l} \eta_{i}^{2} \\
& +\sum_{m=1}^{k} \beta_{i}\left(\sum_{m=1}^{k}\left(y_{i, m}-\omega_{m}^{T} \varphi\left(x_{i}\right)-b_{m}\right)-\eta_{i}\right) \\
& \left.+\sum_{i=1}^{l} \sum_{m=1}^{k} \alpha_{i, m}\left(y_{i, m}-\omega^{T} \varphi\left(x_{i}\right)-b_{m}\right)-\xi_{i, m}\right)
\end{aligned}
$$

The following equations may be got by solving the partial derivatives of $\omega_{m}, b_{m}, \xi_{i, m}, \eta_{i}, \beta_{i}$ and $\alpha_{i, m}$ in Eq. (2) and eliminating $\omega_{m}, b_{m}, \xi_{i, m}$ and $\eta_{i}$.

$$
\left\{\begin{array}{l}
\sum_{i=1}^{l}\left(\beta_{i}+\alpha_{i, m}\right)=0 \\
\sum_{m=1}^{k} \sum_{i=1}^{l} \beta_{i} k_{m}\left(x_{i}, x_{j}\right)+\sum_{m=1}^{k} \sum_{i=1}^{l} \alpha_{i, m} k_{m}\left(x_{i}, x_{j}\right)+\sum_{m=1}^{k} b_{m}+\frac{\beta_{i}}{C_{0}}=\sum_{m=1}^{k} y_{j, m} \\
\sum_{i=1}^{l} \beta_{i} k_{m}\left(x_{i}, x_{j}\right)+\sum_{i=1}^{l} \alpha_{i, m} k_{m}\left(x_{i}, x_{j}\right)+b_{m}+\frac{\alpha_{i, m}}{C_{m}}=y_{j, m}
\end{array}\right.
$$

Where: $\mathrm{j}=1,2, \ldots, 1 ; ; k\left(x, x^{\prime}\right)=\left(\varphi(x) \cdot \varphi\left(x^{\prime}\right)\right)$ represents the kernel function.

$\alpha_{i, m}, \beta_{i}$ and $b_{m}$ may be obtained by solving the above equations.

\section{B. Profile of PSO}

PSO as one smart algorithm put forward by Kennedy and Eberhart in 1995 was spired by the bird flock preying behaviors in nature, where it was thought that birds might exchange information during their preying processes to find out the food positions together. For determining the precise position of foods, each bird necessarily calculated its fitness to estimate its distance to foods; and such information is shared; and then all birds are constantly close to the position of foods and finally find out the accurate position of foods[12-15].

PSO's optimal space search solution processes may be described as follows:

A group of random particles without volume and mass flies in the solution space by a certain mass and speed;

Each particle adjusts the flying speed of the particle swarm based on its comprehensive judgment of flying experience of its own and the overall swarm to continuously update its position and speed; thus, the final target position may be found.

While assuming a swarm including $\mathrm{m}$ particles, Vectors $x_{t}=\left(x_{t 1}, x_{t 2}, \cdots, x_{t m}\right)$ and $v_{t}=\left(v_{t 1}, v_{t 2}, \cdots, v_{t m}\right)$ represent the various particle position and speed at the time $(\mathrm{t})$, respectively. The recursion equations for the position and speed of each particle are as follows:

$$
\begin{aligned}
& v_{t+1}=\omega v_{t}+r_{1} \cdot \operatorname{rand}() \cdot\left(p_{t}-x_{t}\right)+r_{2} \cdot \operatorname{rand}() \cdot\left(g_{t}-x_{t}\right) \\
& x_{t+1}=x_{t}+v_{t}
\end{aligned}
$$

Where: $\omega$ represents the inertia weight of the particle movement; $r_{1}$ and $r_{2}$ represent the acceleration constants and $r_{1}=r_{2}=2$ exists generally to gain a better iteration result; $\operatorname{rand}()$ is to generate a random number evenly distributed in $[0,1] ; p_{t}$ represents the optimal solution for the current particle during the period $(0 \sim t)$; and $g_{t}$ represent the optimal solution for the particle swarm during the period $(0 \sim \mathrm{t})$, which is a global extremum value.

During the calculation processes, $v \in\left[-v_{\max }, v_{\max }\right]\left(v_{\max }\right.$ is a constant) may be specified according to the actual conditions; namely, the speed of any particle is within a certain range to prevent its being out of the solution space.

\section{PSO-MRLSSVM MODEL}

\section{A. Determination of Key Parameters for MRLSSVM}

Actually extends LSSVM to multiple-category dimensions. It may be thought that each dimension has one LSSVM structure. In contrast, constraints shall be necessarily satisfied but the fitting error in a single dimension shall be minimum; on the other hand, the total of the fitting errors in all dimensions shall be minimum. As for any algorithm including LSSVM structures, optimization of those key parameters is crucial for influencing the SVM's performances for any specific issue.

MRLSSVM's objective function is as follows:

$$
\min \frac{1}{2} \sum_{m=1}^{k}\left\|\omega_{m}\right\|^{2}+\frac{1}{2} C_{0} \sum_{i=1}^{l} \eta_{i}^{2}+\frac{1}{2} \sum_{i=1}^{l} \sum_{m=1}^{k} C_{m} \xi_{i, m}^{2}
$$

Where: $C_{0}$ represents the penalty parameter of the overall samples; $C_{m}$ represents the penalty parameter of each dimension; and $m$ represents the dimensions of outputs of the model.

The radial basis kernel function was selected here as the kernel function of our MRLSSVM, which is expressed by:

$$
K\left(x_{i}, x_{j}\right)=\exp \left[-\left|x_{i}-x_{j}\right|^{2} / \delta^{2}\right]
$$

Where: $\delta$ (kernel function radius) represents the unknown parameters and each dimension corresponds to its own kernel function radius $\left(\delta_{i}\right)$.

On the whole, key parameters for optimization include: $C_{0}$, $C_{1}, C_{2} \ldots, C_{\mathrm{m}}$ and $\delta_{1}, \delta_{2}, \ldots, \delta_{m}$. Namely, there are $(2 \mathrm{~m}+1)$ key parameters for any $\mathrm{m}$-dimensional issue. Thus, an appropriate method is necessary to determine these key parameters.

\section{B. Design of a PSO-MRLSSVM Model}

With reference to the thought for optimization of parameters for LSSVM, PSO is adopted to optimize key parameters for MRLSSVM which is schematically shown in Figure 1; and the detailed steps are as follows:

Step 1: Initialization of the particle swarm: the dimensions (D) of particles are determined; namely, parameters for optimization by means of MRLSSVM include $C_{0}, C_{m}$ and $\delta_{m}$. The size of population of particles $(N)$, the position and speed 
of each particle, $C_{1}$ and $C_{2}$, and $\omega_{\max }$ and $\omega_{\min }$ are determined; and the maximum number of iterations $\left(k_{\max }\right)$ is set; in addition, the current number of iterations $(k)$ is initialized to be 1 .

Step 2: Calculation of the fitness: the coordinates of the corresponding position of the $i^{\text {th }}$ particle are taken as the parameter set for training MRLSSVM; and the fitness of the $i^{\text {th }}$ particle $(F i t(i))$ is calculated.

Step 3: Evaluation of particles: Fit $(i)$ and the individual extremum value (pbest $\left.t_{\mathrm{i}}\right)$ are compared; if Fit $(i)>$ pbest $_{\mathrm{i}}$, Fit $(i)$ Fit $(i)$ replaces pbest $t_{1}$; moreover, Fit $(i)$ and the global extremum value (gbest) are compared; if Fit( $(i)>$ gbest, Fit $(i)$ replaces gbest.

Step 4: Updating position and speed of each particle: the speed and position of each particle are updated in accordance with Eq. (4).

Step 5: Judgment of iterations may be terminated: if the current number of Iterations is up to the preset $k_{\max }$, iterations shall be terminated for outputting the optimal solution of parameters; at the same time, the trained MRLSSVM is the optimal algorithm model; otherwise, the current number of Iterations (k) increases by 1 , Steps 2 4 are performed again.

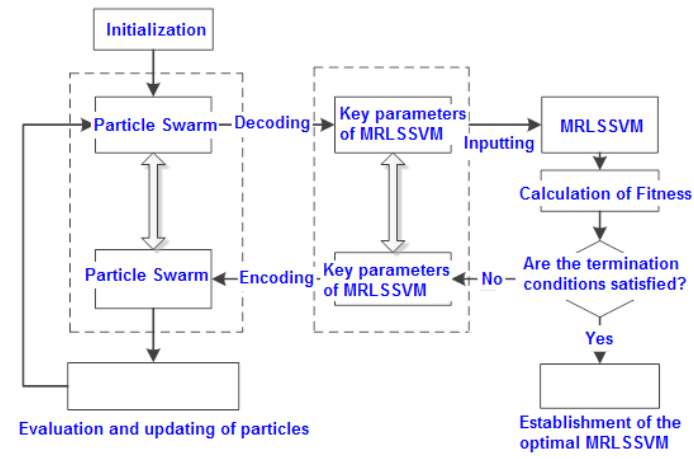

FIGURE I. FLOW DIAGRAM OF SOLUTION OF KEY PARAMETERS FOR PSO-MRLSSVM

\section{SimUlation TESTS AND APPLICATION EXAMPLES}

\section{A. Description of Tests}

Our data were collected by means of a ZT-3 type multifunctional rotor fault simulation test bench (manufacturer: Nanjing SEU Vibrometer Factory) where various conventional faults may be simulated for the power rotor system and whose structural representation is shown in Figure 2. Normal and fault vibration signals were sampled in the rotor test bench. The sampling frequency is $2048 \mathrm{~Hz}$; and the rotor speed is $6000 \mathrm{rpm}$. Collected analog data were synchronously converted into digital signals by means the $\mathrm{A} / \mathrm{D}$ converter to input them to a computer. Several faults were simulated, which are as follows:

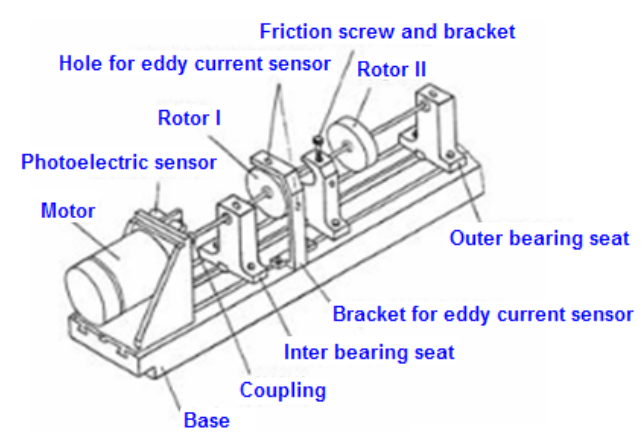

FIGURE II. STRUCTURAL REPRESENTATION OF ZT-3 TYPE MULTIFUNCTIONAL ROTOR FAULT SIMULATION TEST $\mathrm{BENCH}$

Single fault types: rotor imbalance, rotor mal-alignment, bearing inner ring damage and bearing outer ring damage;

Concurrent fault types: imbalance-mal-alignment, imbalance - inner ring damage, imbalance - outer ring damage.

There are 4 types of single faults:

- 1. Rotor imbalance

- 2. Rotor mal-alignment

- 3. Bearing inner ring damage

- 4. Bearing outer ring damage

In addition, there are concurrent fault types of the above 4 types of single faults. While the above 4 types of single faults are encoded as $d_{1}, d_{2}, d_{3}$ and $d_{4}$ and two types of single faults occur at the same time, $d_{12}$ may represent for short concurrence of $\mathrm{d}_{1}$ and $\mathrm{d}_{2}$ (namely rotor imbalance - mal-alignment. Any normal type is regarded as a special fault type which is encoded as $d_{0}$.All fault types and corresponding codes are presented in Table 1.

TABLE I. FAULT TYPES AND CORRESPONDING CODES

\begin{tabular}{|c|l|}
\hline Fault Code & \multicolumn{1}{|c|}{ Fault Type } \\
\hline$d_{0}$ & Normal operation \\
\hline$d_{1}$ & Rotor imbalance \\
\hline$d_{2}$ & Rotor mal-alignment \\
\hline$d_{3}$ & bearing inner ring damage \\
\hline$d_{4}$ & bearing outer ring damage \\
\hline$d_{12}$ & rotor imbalance + rotor mal-alignment \\
\hline$d_{13}$ & Rotor imbalance + bearing inner ring damage \\
\hline$d_{14}$ & Rotor imbalance + bearing outer ring damage \\
\hline$d_{123}$ & $\begin{array}{l}\text { Rotor imbalance + rotor mal-alignment + bearing inner } \\
\text { ring damage }\end{array}$ \\
\hline$d_{124}$ & $\begin{array}{l}\text { Rotor imbalance + rotor mal-alignment + bearing outer } \\
\text { ring damage }\end{array}$ \\
\hline Experiments were carried out for comparison
\end{tabular}
MRLSSVM and PSO-MRLSSVM classifiers; and the 5-fold cross validation method was used. 200 feature data of various fault types were selected, which were divided evenly into 5 subsets ( 1 subset for testing and 4 sunsets for training). Each model was trained for 5 times, respectively; and 5 diagnosis rates were correspondingly gained, which were averaged finally as the fault diagnosis rate of the model. The distribution of data sample sets is shown in Table 2. 
TABLE II. DISTRIBUTION OF DATA SAMPLE SETS

\begin{tabular}{|c|l|l|l|}
\hline & \multicolumn{1}{|c|}{ Single fault } & \multicolumn{1}{|c|}{ Concurrent fault } & \multicolumn{1}{c|}{ Total } \\
\hline Training set & $800\left(5^{*} 160\right)$ & $800\left(5^{*} 160\right)$ & 1600 \\
\hline Testing set & $200\left(5^{*} 40\right)$ & $200\left(5^{*} 40\right)$ & 400 \\
\hline Total & 1000 & 1000 & 2000 \\
\hline
\end{tabular}

There are 10 groups of fault types of training sets (single and concurrent faults) in total to be input into MRLSSVM; and then classifiers were tested by various fault types of testing sets. Table 3 presents outputs for two types of classifiers as for several groups of the same measurement samples.

TABLE III. FAULT OUTPUTS

\begin{tabular}{|c|c|c|c|}
\hline $\begin{array}{l}\text { Fault } \\
\text { type }\end{array}$ & $\begin{array}{c}\text { Fault } \\
\text { classification } \\
\text { model }\end{array}$ & Actual output & $\begin{array}{l}\text { Expected } \\
\text { output }\end{array}$ \\
\hline \multirow{2}{*}{$d_{12}$} & MRLSSVM & $\begin{array}{l}{[0.0527,0.9142,0.9314,} \\
0.1553,0.1866]\end{array}$ & \multirow{2}{*}[0,1,1,0,0]{} \\
\hline & PSO-MRLSSVM & $\begin{array}{l}{[0.0376,0.9425,0.9287,} \\
0.1487,0.1643]\end{array}$ & \\
\hline \multirow{2}{*}{$d_{123}$} & MRLSSVM & $\begin{array}{l}{[0.0127,0.7042,0.8414,} \\
0.6833,0.1466]\end{array}$ & \multirow{2}{*}[0,1,1,1,0]{} \\
\hline & PSO-MRLSSVM & $\begin{array}{l}{[0.1076,0.7614,0.8101,} \\
0.7854,0.2143]\end{array}$ & \\
\hline \multirow{2}{*}{$d_{124}$} & MRLSSVM & $\begin{array}{l}{[0.2127,0.6842,0.6988,} \\
0.3540,0.7466]\end{array}$ & \multirow{2}{*}[0,1,1,0,1]{} \\
\hline & PSO-MRLSSVM & $\begin{array}{l}{[0.1156,0.8325,0.7544,} \\
0.2487,0.6954]\end{array}$ & \\
\hline
\end{tabular}

A threshold $(\lambda)$ was selected to classify the fault types and gain the diagnosis results. While the output of a certain fault type is more than $\lambda$, it is thought that such type of faults exist. The range of $\lambda$ was determined among $[0.1,0.9]$, whose initial value is 0.1 ; and its growth step is 0.1 in sequence. 9 thresholds and 3 classifiers were tested to determine the corresponding diagnosis rates. As for each threshold, the diagnosis rates for 10 independent experiments were averaged as the diagnosis rate of the current classification model under this threshold. The fault diagnosis rate for each group of experiments is the diagnosis rate obtained by means of the 5 -fold cross validation method. The measurement data covered all data samples. The model diagnosis rates are listed in Table 4.

TABLE IV. MODEL DIAGNOSIS RATE STATISTICAL TABLE

\begin{tabular}{|c|c|c|c|c|c|}
\hline$\lambda$ & \multirow{2}{*}{$\begin{array}{c}\text { Total } \\
\text { samples }\end{array}$} & \multicolumn{4}{|c|}{ Fault diagnosis model } \\
\cline { 3 - 6 } & & \multicolumn{2}{|c|}{ MRLSSVM } & \multicolumn{2}{c|}{ PSO-MRLSVM } \\
\cline { 3 - 6 } & & $\begin{array}{c}\text { Number of } \\
\text { diagnosis } \\
\text { successes }\end{array}$ & $\begin{array}{c}\text { Diagnosis } \\
\text { Rate (\%) }\end{array}$ & $\begin{array}{c}\text { Number of } \\
\text { diagnosis } \\
\text { successes }\end{array}$ & $\begin{array}{c}\text { Diagnosis } \\
\text { Rate (\%) }\end{array}$ \\
\hline 0.1 & $2000 * 10$ & 7438 & 37.19 & 9008 & 45.04 \\
\hline 0.2 & $2000 * 10$ & 11214 & 56.07 & 12480 & 62.40 \\
\hline 0.3 & $2000 * 10$ & 14200 & 71.00 & 14265 & 71.33 \\
\hline 0.4 & $2000 * 10$ & 14425 & 72.13 & 14684 & 73.42 \\
\hline 0.5 & $2000 * 10$ & 16003 & 80.02 & 16809 & 84.05 \\
\hline 0.6 & $2000 * 10$ & 15671 & 78.36 & 17078 & 85.39 \\
\hline 0.7 & $2000 * 10$ & 14483 & 72.42 & 16004 & 80.02 \\
\hline 0.8 & $2000 * 10$ & 13225 & 66.13 & 14648 & 73.24 \\
\hline 0.9 & $2000 * 10$ & 9828 & 49.14 & 10060 & 50.30 \\
\hline
\end{tabular}

Table 4 indicates that the fault diagnosis rate is affected greatly by $\lambda$; a too large or low threshold may lead to no correct identification of any fault type; thus, the diagnosis rate falls. While $\lambda$ is taken as 0.1 or 0.9 , the diagnosis success rates for two models are relatively low. As for MRLSSVM, the diagnosis rate rises along with the growth of $\lambda(\lambda \in[0.1,0.5])$; on the other hand, the diagnosis rate falls along with the growth of $\lambda(\lambda \in[0.5,0.9])$; in addition, the diagnosis rate peaks $(80.02 \%)$ while $\lambda=0.5$; thus, MRLSSVM may be utilized to well classify fault diagnosis types for rotary machines and it is effective for concurrent fault diagnosis. On the other hand, the fault diagnosis rate peaks $(85.39 \%)$ while $\lambda=0.6$ as for PSOMRLSSVM. While the threshold is the same, the fault diagnosis rate is always larger for PSO-MRLSSVM rather than MRLSSVM. Thus, the optimal parameter set is more easily searched by means of our PSO-MRLSSVM so that the classification precision may be improved and its effectiveness shall be verified.

\section{CONCLUSIONS}

In view of those characteristics such as several outputs of any concurrent fault for rotating machines, MRLSSVM was applied here for diagnosis of mechanical faults. Experimental results indicate that mechanical faults may be well classified by means of such algorithm

Then, PSO was utilized to optimize the key parameters for MRLSSVM to solve those issues (such as there being many key parameters and aimless determination of parameters) for MRLSSVM. Experimental results verify our method shall be feasible and effective.

\section{REFERENCES}

[1] Dou Dongyang, Zhao Yingkai. A Priority and Diagnosis Tree-based Expert System for Fault Diagnosis of Rotating Machinery [J]. Proceedings of the CSEE, 2008 (32): 82-88.

[2] Feng Ke, Cui Yonggu, Li Jing, Ni Chengjun. Fault diagnosis based on fuzzy logic and genetic algorithms of engineering machine [J]. Journal of PLA University of Science and Technology (Natural Science Edition), 2006 (04): 385-389.

[3] Yu Yuan, Xing Zhao, Jiyou Fei, Yulong Zhao, Jiahui Wang, Jiawei Xiang. Study on Fault Diagnosis of Rolling Bearing Based on TimeFrequency Generalized Dimension [J]. Shock and Vibration, 2015: 2015

[4] Li Zhiliang, Xu Xiaobin, Wen Chenglin. A New Approach of Simultaneous Faults Diagnosis Based on DSmT [J]. Journal of Hangzhou Dianzi University (Natural Sciences), 2008 (06): 107-110.

[5] Guo Qing, Xia Hong, Han Wenwei. Application Research of DSmT Fusion Algorithm Based on Concurrent Fault Information [J]. Journal of Hunan University (Natural Sciences), 2015 (02): 35-39+59.

[6] Yuming Chen, Yongzhi Li, Yingkai Zhao. Sub-pixel detection algorithm based on cubic B-spline curve and multi-scale adaptive wavelet transform [J]. Optik - International Journal for Light and Electron Optics, 2016, 127(1):

[7] Li Ruitong, Wang Huaqing, et al. Application of CICA in Compound Fault Feature Extracting of Rolling Bearings [J]. Noise and Vibration Control, 2015 (03): 173-176.

[8] Chatterjee A, Siarry P. Nonlinear inertia weight variation for dynamic adaptation in particle swarm optimization [J]. Computers and Operations Research, 2006,33(3): 859-871.

[9] Dongying Han, Pei li, Shujun An,Peiming Shi. Multi-frequency weak signal detection based on wavelet transform and parameter compensation band-pass multi-stable stochastic resonance [J]. Mechanical Systems and Signal Processing, 2016, 70-71:.

[10] Teng Zhongpei, Gou Yi. The Research of Motor Faulty Diagnosis in Multi-fault Coupling Mode Based on the Artificial Immunity System [J]. Modern Machinery, 2009, (05): 4-6+12.

[11] $\mathrm{Hu}$ Changhua, Cai Yanning, Zhang Qi. Simultaneous fault diagnosis based on multi-regression LSSVM [J]. Journal of Huazhong University of Science and Technology (Natural Science Edition), 2009, (S1): 1-5.

[12] Zhang Qinghua, Qianyu, et al. Study of integrated fault diagnosis technologies based on the artificial immunity system [J]. Noise and Vibration Control, 2008, (03): 56-59. 
[13] Wang Junwei, Wang Dingwei. Experiments and analysis on inertia weight in particle swarm optimization [J]. Journal of Systems Engineering, 2005, 20 (2): 195-199.

[14] Siliang Lu, Qingbo He, Haibin Zhang, Fanrang Kong. Rotating machine fault diagnosis through enhanced stochastic resonance by full-wave signal construction [J]. Mechanical Systems and Signal Processing, 2016.

[15] Sun Weichao, Li Wenhai, Li Wenfeng. Avionic devices fault diagnosis based on fusion method of rough set and D-S theory [J]. Journal of Beijing University of Aeronautics and Astronautics, 2015, (10): 19021909. 